\title{
Efeitos preventivos e terapêuticos do exercício físico sobre lesão pulmonar e estresse oxidativo induzido por bleomicina
}

\author{
Preventive and therapeutic effects of physical exercise \\ on bleomycin-induced lung injury and oxidative stress
}

\author{
Ricardo Aurino de Pinho \\ Fernanda Schuveitzer Soares \\ Luís Gustavo da Costa Rocha \\ Cleber Aurino de Pinho ${ }^{1}$ \\ Luciano Acordi da Silva \\ Paulo César Look da Silveira
}

1 Universidade do Extremo Sul Catarinense. Laboratório de Fisiologia e Bioquímica do Exercício. Programa de Pós-Graduação em Ciências da Saúde. Criciúma, SC. Brasil.

Recebido em 31/05/08 Revisado em 05/11/08 Aprovado em 13/02/09
Resumo - Estudos têm apontado o exercício físico regular de baixa a moderada intensidade como um importante agente no combate ao estresse oxidativo pulmonar. Esse estudo investigou o efeito preventivo e terapêutico do exercício físico na resposta oxidativa pulmonar induzida por Bleomicina (BLM). Métodos: 36 camundongos machos (CF1, 30-35g) foram divididos em 6 grupos $(n=6)$ : não treinados salina ou BLM, treinamento preventivo salina ou BLM, treinamento terapêutico salina ou BLM e receberam endotraquealmente uma única dose de BLM $(2,5 \mathrm{U} / \mathrm{Kg}$ em $0,25 \mathrm{~mL}$ de soro estéril) ou salina $(0,9 \%)$. Os grupos treinados realizaram um programa de exercício progressivo em esteira durante 8 semanas (até $17 \mathrm{~m}$.min-1, 50min.dia-1). O grupo preventivo iniciou o programa de exercício 62 dias antes da administração de BLM e o grupo terapêutico 62 dias após a administração de BLM. Quarenta e oito horas após o período experimental, todos os animais foram mortos por decapitação e o pulmão direito foi cirurgicamente removido. Foi determinado o conteúdo de hidroxiprolina, os níveis de TBARS e carbonilação de proteínas, atividades da superóxido dismutase e catalase. Os resultados mostram que os treinamentos preventivo e terapêutico reduzem significativamente o conteúdo de hidroxiprolina e inibem o aumento nos marcadores de danos oxidativos em lipídeos e proteínas. Contudo, somente o treinamento terapêutico reduziu a atividade da SOD e catalase nos animais expostos a BLM. A realização de treinamento físico antes ou após da lesão pulmonar é um agente capaz de minimizar o estresse oxidativo induzido pela BLM.

Palavras-chave: Exercício físico, estresse oxidativo, pulmão, bleomicina, radicais livres.

Abstract - Studies have shown that regular physical exercise of moderate intensity is an important tool for the control of pulmonary oxidative stress. The objective of this study was to examine the preventive and therapeutic effect of physical exercise on oxidative stress in the lungs of mice exposed to bleomycin (BLM). Thirty-six male mice (CF1, 30-35 g) received a single endotracheal dose of BLM (2.5 U/kg body weight dissolved in $0.25 \mathrm{~mL} 0.9 \% \mathrm{NaCl})$ or saline $(0.9 \% \mathrm{NaCl})$ and were divided into six groups ( $n=6)$ : untrained saline or BLM, preventive training saline or BLM, and therapeutic training saline or BLM. The trained groups underwent a program of progressive exercise on a treadmill for 8 weeks (up to $17 \mathrm{~m} \cdot \mathrm{min}^{-1}, 50 \mathrm{~min}^{-d a y^{-1}}$ ). The preventive group started the exercise program 62 days before the administration of BLM and the therapeutic group 62 days after the administration of BLM. All animals were killed by decapitation 48 hours after the experimental period, and the right lung was surgically removed for the determination of biochemical parameters. Hydroxyproline content, TBARS level, protein carbonylation, and superoxide dismutase (SOD) and catalase (CAT) activities were analyzed. The results showed that preventive and therapeutic training led to a significant reduction in hydroxyproline content and inhibited the increase in oxidative damage to lipids and proteins. However, only therapeutic training decreased SOD and CAT activities in mice exposed to BLM. The results suggest that preventive and therapeutic physical exercise is able to minimize pulmonary oxidative stress induced by BLM.

Key words: Physical exercise; Oxidative stress; Lung; Bleomicyn; Free radical 


\section{INTRODUÇÃO}

Para melhor compreender as respostas pulmonares estruturais e bioquímicas da lesão pulmonar, diversos estudos têm utilizado a administração de bleomicina (BLM) em ratos, como modelo específico de Fibrose Pulmonar (FP) ${ }^{1-5}$.

A BLM é um dos agentes quimioterápicos utilizado no tratamento inicial de linfomas, tumores testiculares e carcinoma de células escamosas. Dependendo da dose, seu uso em humanos pode produzir, ao longo do tempo, uma série de lesões associadas com as mudanças funcionais e bioquímicas da FP, processos inflamatórios e infiltração de células inflamatórias, aumento do conteúdo de colágeno, e redução da capacidade e volume pulmonar .

É possível que os danos pulmonares causados pela BLM estejam diretamente relacionados à formação de Espécies Reativas de Oxigênio (ERO) por um mecanismo dependente de ferro ${ }^{7}$. Ademais, a produção de ERO pode aumentar o dano pulmonar por inativação de antiproteases, rompimento de membranas e danos alveolares ${ }^{8,9}$.

Um eficiente sistema de defesa pode minimizar o dano oxidativo pulmonar induzido pela BLM. Esse sistema de defesa pode atuar de forma associada ou independente pela ativação de enzimas antioxidantes ou pela ação de antioxidantes biológicos não-enzimáticos ${ }^{10,11}$.

Adicionalmente, agentes terapêuticos podem contribuir para prevenir ou reverter os danos oxidativos causados pelo excesso de ERO, dentre os quais, destaca-se o exercício físico. Além de seus efeitos sobre os sistemas antioxidantes, o exercício físico regular exerce um efeito positivo sobre a capacidade cardiorrespiratória e sobre a resposta bioquímica pulmonar ${ }^{12}$. Estudos anteriores ${ }^{10,12,13}$ mostram que a realização de exercícios físicos regulares como mecanismo terapêutico no tratamento de doenças ou lesão reduz a produção de oxidantes, os danos oxidativos e melhoram o sistema de defesa antioxidante pulmonar. Entretanto, ainda não está claro se o exercício físico promove uma proteção pulmonar contra estresse oxidativo.

Diversos estudos têm descrito as vias metabólicas da produção das ERO durante o exercício ${ }^{14-16}$, porém pouco se conhece sobre a influência do exercício físico regular sobre a liberação de ERO induzida por agentes químicos, especificamente, a BLM. As informações na literatura são apenas pressupostos que necessitam de investigação científica mais apurada.

Assim, o objetivo desse estudo foi investigar o efeito preventivo ou terapêutico do exercício físico regular na resposta oxidativa pulmonar induzida por BLM. Portanto, nossa hipótese é que os parâmetros de estresse oxidativo pulmonar, induzidos pela BLM, possam ser minimizados de forma preventiva ou terapêutica, após a realização de um programa de exercício físico. Acredita-se que elucidar esse aspecto é de extrema relevância para uma melhor compreensão do fenômeno bioquímico dos danos oxidativos pulmonares e de sua relação com o exercício físico.

\section{PROCEDIMENTOS METODOLÓGICOS}

Todos os procedimentos foram realizados de acordo com "Guiding Principles in the Care and uses of Animals"17 e aprovados pelo Comitê de Ética da Universidade do Extremo Sul Catarinense, conforme parecer 026/2007.

Animais: 36 camundongos machos (CF1), com idade de 8 semanas e peso de $30-35 \mathrm{~g}$, foram divididos randomicamente em 6 grupos $(\mathrm{n}=6)$ : não treinados salina, não treinados bleomicina, treinamento preventivo salina, treinamento preventivo bleomicina, treinamento terapêutico salina, treinamento terapêutico bleomicina. Os animais foram alocados num ambiente com temperatura controlada a $23^{\circ} \mathrm{C}$, num ciclo de iluminação de 12 horas claro/escuro e com alimentação/hidratação ad libitum.

Modelo de lesão pulmonar por bleomicina: os animais receberam endotraquealmente uma única dose de BLM (2,5 U/Kg de massa corporal dissolvida em 0,25mL de soro estéril 0,9\%) conforme descrito por $^{2}$. Os animais controles foram submetidos ao mesmo protocolo, porém receberam somente a administração de soro estéril 0,9\%. A instilação de BLM foi feita sob anestesia (cetamina, $80 \mathrm{mg} / \mathrm{Kg}$ de massa corporal, ip) e relaxante muscular (xilazina, $20 \mathrm{mg} / \mathrm{Kg}$ de massa corporal, ip).

Protocolo de exercício: todos os animais foram ambientados em uma esteira ergométrica (10 m.min ${ }^{-1}$ sem inclinação, 10 min.dia ${ }^{-1}$ ) durante uma semana. Após esse período, os grupos treinados foram submetidos a um programa de treinamento progressivo, 5 vezes por semana, durante 8 semanas, com velocidade entre $12 \mathrm{~m} \cdot \mathrm{min}^{-1} \mathrm{e} 20 \mathrm{~m} \cdot \mathrm{min}^{-1}$, tempo até 50 min.dia ${ }^{-1}$, sem inclinação e sem receberem estímulos nocivos. Segundo Fernando et al..$^{18}$, a velocidade utilizada neste protocolo representa um consumo máximo de oxigênio de 74\% a 85\%.

Desenho Experimental: para o treinamento preventivo, os animais foram submetidos ao programa de exercício físico e 48 horas após o treinamento, receberam a administração de BLM ou salina, permanecendo em repouso por 60 dias. No treinamento 
terapêutico, os animais receberam, inicialmente, a administração de BLM ou salina, permanecendo em repouso por 60 dias. Quarenta e oito horas após o período de repouso, os animais foram submetidos ao programa de exercício físico. $\mathrm{O}$ grupo não treinado permaneceu por 120 dias em repouso.

Após o período experimental, os animais foram mortos por deslocamento cervical e o pulmão direito foi retirado cirurgicamente, processado, aliquotado e imediatamente, armazenado a $-70^{\circ} \mathrm{C}$ para posteriores análises.

Conteúdo de Hidroxiprolina: o conteúdo de hidroxiprolina no tecido pulmonar foi usado como índice quantitativo de fibrogênise. Foi determinado espectrofotometricamente a $550 \mathrm{~nm}$ como previamente descrito por ${ }^{19}$ e expresso em miligrama de hidroxiprolina por miligrama de tecido.

Danos oxidativos: como índice de peroxidação de lipídeos, foi determinado a formação de substâncias reativas ao aquecimento do ácido tiobarbitúrico medido espectrofotometricamente $(532 \mathrm{~nm})$ e expressos em nmol/mg de proteína ${ }^{20}$. Os danos oxidativos em proteínas foram mensurados pela determinação de grupamento carbonil baseados na reação com dinitrofenilhidrazina ${ }^{21}$. O conteúdo de carbonil foi determinado espectrofotometricamente em 370nm, usando um coeficiente 22.0000 Molar e expresso em nmol/mg de proteína.

Enzimas antioxidantes: a atividade da Superóxido Dismutase (SOD) foi determinada pela inibição da auto-oxidação da adrenalina medida espectrofotometricamente (480 nm) e expressa em U SOD/mg de proteína ${ }^{22}$. A atividade da Catalase (CAT) foi determinada pela queda na absorbância correspondente ao consumo peróxido de hidrogênio, medida espectrofotometricamente (240nm) e expressa em U CAT/mg de proteína ${ }^{23}$.

Determinação de Proteínas: a quantidade total de proteína foi determinada a partir da técnica de Lowry et al. ${ }^{24}$.

Análise Estatística: os dados foram expressos em média e erro padrão médio, analisados estatisticamente pelo teste $\mathrm{t}$ de student. $\mathrm{O}$ nível de significância estabelecido foi de $\mathrm{p}<0,05$. Foi utilizado o SPSS (Statistical Package for the Social Sciences) versão 15.0 como pacote estatístico.

Reagentes: Ácido tiobarbitúrico, DTNB, dinitrofenilhidrazina, adrenalina e peróxido de hidrogênio, 3,3',5,5'-tetrametilbenzidine, dimetilaminobenzladeido, foram adquiridos da Sigma Chemical Co. (St. Louis, MO). 2,2'-azobis (2-metilpropionamidina) diidroclorido foi adquirido da Aldrich Chemical (Milwaukee, WI).

\section{RESULTADOS}

Conteúdo de hidroxiprolina: conforme figura 1 , os resultados mostram um aumento significativo no conteúdo de hidroxiprolina, 60 dias após a administração de BLM. Contudo, os animais que foram submetidos ao treinamento físico preventivo e terapêutico tiveram níveis menores em relação aos grupos não-treinados e não alteraram esses valores em relação ao grupo salina.

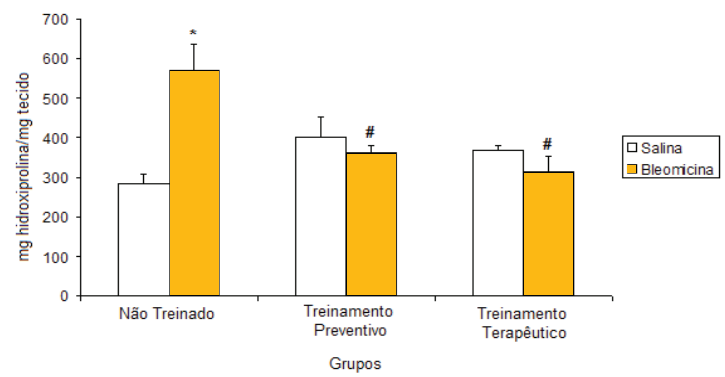

Figura 1. Efeitos do treinamento físico preventivo e terapêutico sobre o conteúdo de hidroxiprolina nos pulmões de camundongos expostos a Bleomicina. Os valores são apresentados em Média土EPM e os resultados são expressos em nmol de TBARS/mg de proteína. A diferença significativa no grupo não treinado(*) e nos grupos treinados em relação ao não treinado ${ }^{(\#)}$ foi de $p<0,05$.

Danos oxidativos: Os resultados apresentados na figura 2 mostram um aumento significativo nos níveis TBARS no grupo tratado com BLM. Os grupos treinados antes e após a lesão por BLM não alteraram os níveis de TBARS, em relação ao grupo salina e ao grupo não-treinado. Similarmente, a figura 3 mostra um aumento nos níveis de carbonilação de proteínas no grupo tratado com BLM. No entanto, os grupos treinados salina tiveram um aumento significativo em relação ao grupo salina não-treinado.

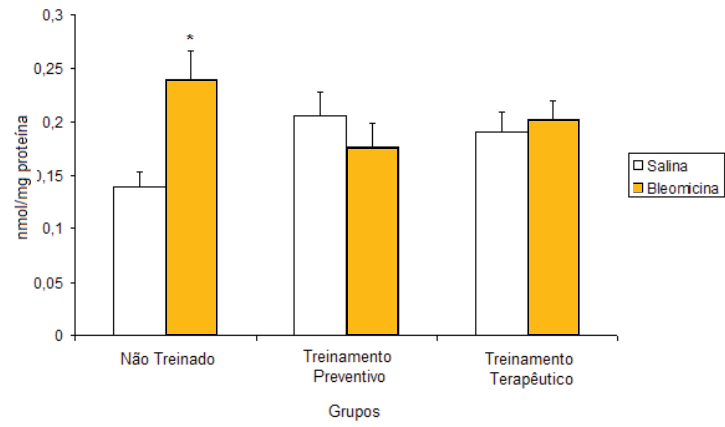

Figura 2. Efeitos do treinamento físico preventivo e terapêutico sobre os níveis de TBARS em pulmões de camundongos expostos a Bleomicina. Os valores são apresentados em Média EPMM e os resultados são expressos em nmol de TBARS/ mg de proteína. A diferença significativa no grupo não treina$\mathrm{do}^{(*)}$ e nos grupos treinados em relação ao não treinado ${ }^{(\#)}$ foi de $p<0,05$. 


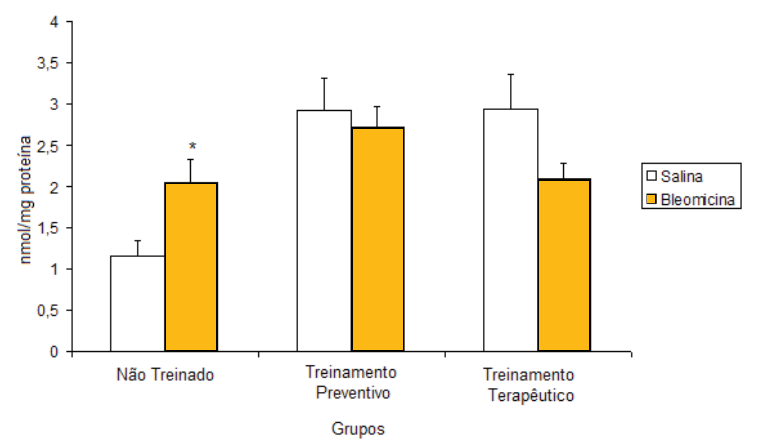

Figura 3. Efeitos do treinamento físico preventivo e terapêutico sobre os níveis de carbonilação de proteínas em pulmões de camundongos expostos a Bleomicina. Os valores são apresentados em Média \pm EPM e os resultados são expressos em nmol/mg de proteína. A diferença significativa no grupo não treinado ${ }^{(*)}$ e nos grupos treinados em relação ao não treinado ${ }^{(\#)}$ foi de $p<0,05$.

Enzimas antioxidantes: os resultados da figura 4 mostram uma menor atividade da SOD dos animais tratados com BLM, no grupo não-treinado e treinamento terapêutico, enquanto que os animais que receberam salina apresentaram uma maior atividade. Porém, no grupo com treinamento preventivo, a atividade da SOD foi aumentada somente no grupo tratado com BLM. Na atividade da CAT, os resultados apresentados na figura 5 mostram uma diminuição significativa tanto nos animais que receberam salina e BLM quanto nos grupos preventivo e terapêutico.

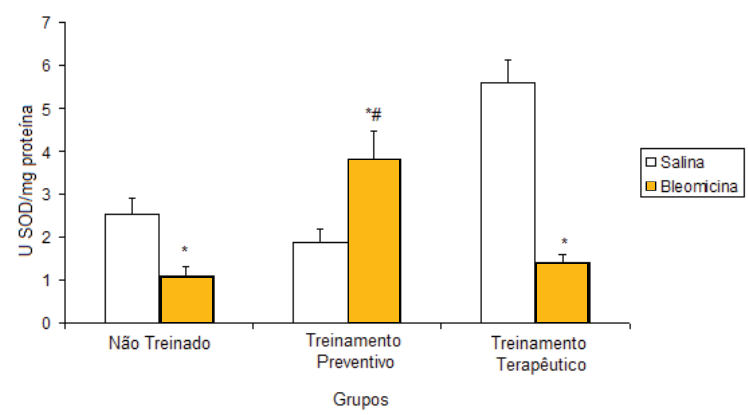

Figura 4. Efeitos do treinamento físico preventivo e terapêutico sobre a atividade da Superóxido Dismutase em pulmões de camundongos expostos a Bleomicina. Os valores são apresentados em Média \pm EPM e os resultados são expressos em U SOD/mg de proteína. A diferença significativa no grupo não treinado(*) e nos grupos treinados em relação ao não treinado(\#) foi de $p<0,05$.

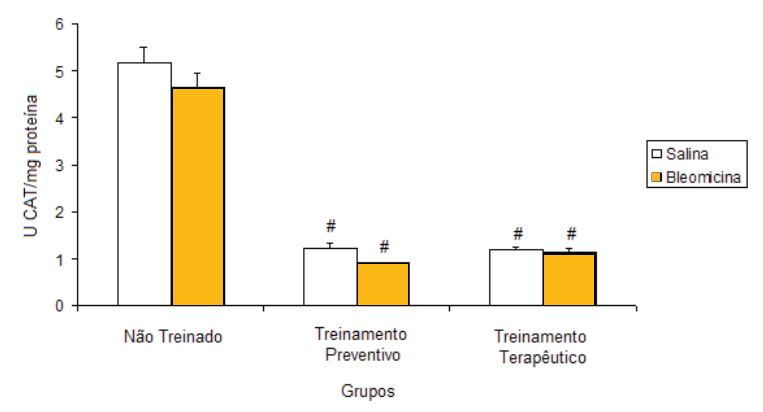

Figura 5. Efeitos do treinamento físico preventivo e terapêutico sobre a atividade da Catalase em pulmões de camundongos expostos a Bleomicina. Os valores são apresentados em Média \pm EPM e os resultados são expressos em U CAT/mg de proteína. A diferença significativa no grupo não treinado ${ }^{(*)}$ e nos grupos treinados em relação ao não treinado ${ }^{(\#)}$ foi de $p<0,05$.

\section{DISCUSSÃO}

Oxercício físico regular tem sido usado como recurso terapêutico auxiliar em diversas doenças crônico-degenerativas. Contudo, pouco se conhece sobre os efeitos do exercício físico realizado de forma preventiva ou terapêutica sobre os marcadores de estresse oxidativo pulmonar induzidos por agentes químicos, especificadamente, a BLM .

Inicialmente, nós demonstramos que o uso agudo da BLM provoca alterações nos níveis de hidroxiprolina, indicando um aumento no conteúdo de colágeno e um processo de fibrogênese pulmonar (figura 1). Esses resultados estão de acordo com outros estudos $2,25-27$. Adicionalmente, demonstramos, ainda, que o exercício físico realizado tanto preventivamente quanto de forma terapêutica impede esse aumento.

Os mecanismos fisiopatológicos das lesões pulmonares por BLM não estão bem estabelecidos na literatura, porém evidências mostram que a lesão pulmonar por agentes químicos desencadeia uma resposta inflamatória primária, que resulta, ao longo do tempo, num aumento da produção de $\mathrm{ERO}^{5,27,28 .}$

Adicionalmente, a BLM tem uma fácil interação com o íon $\mathrm{Fe}^{2+}$ que se liga ao DNA. Esse complexo $\mathrm{BLM}-\mathrm{Fe}^{2+}$ reduz o oxigênio do meio e forma ERO, o que, possivelmente, esteja mediando a formação de fibrose ${ }^{3}$. Portanto, ambos os mecanismos de produção de ERO induzidos pela BLM pode aumentar o dano pulmonar por mediar a liberação de fatores pró-inflamatórios, inativação de anti-proteases, síntese de matriz extracelular, aumento no conteúdo de colágeno e proliferação de fibroblastos ${ }^{8}$.

Durante a resposta inflamatória pulmonar, estimulada por agentes químicos como a BLM, neutrófilos e macrófagos migram para o espaço aéreo, os quais estimulam a liberação de proteases e a produção de $\mathrm{ERO}^{28}$. Esse processo gera uma série de reações que culminam na proliferação de fibroblastos e consequente fibrose ${ }^{29}$. É provável que o ânion superóxido seja a principal ERO produzida durante esse processo por estimular os fibroblastos a secretarem colágeno ${ }^{30}$. Portanto, antioxidantes, como a SOD, têm um importante papel no sistema de defesa pulmonar contra a lesão causada pela BLM. Assim, acredita-se que a redução no conteúdo de hidroxiprolina induzido pelo exercício físico seja 
decorrente da melhora do sistema antioxidante estimulado pelo exercício, impedindo que alterações morfológicas e estruturais aconteçam.

No presente estudo, hipotetizamos que a prática regular de exercícios físicos possa inibir ou minimizar os danos oxidativos em proteínas e lipídios de membrana causados pela exposição de BLM.

A lipoperoxidação resulta da reação de radicais livres com ácidos graxos polinsaturados presentes em lipoproteínas de membranas. Mudanças nos marcadores de lipoperoxidação são evidências mais frequentes observadas no tecido pulmonar após exposição à BLM $^{3,4,27,32}$ e na resposta ao exercício físico ${ }^{12,13}$.

Os resultados obtidos em nosso estudo mostram um aumento significativo nos níveis de TBARS, no grupo tratado com BLM. Os grupos treinados antes e após a lesão por BLM não alteraram esses níveis em relação ao grupo salina e ao grupo nãotreinado (figura 2).

É possível que esses resultados sejam decorrentes do aumento nos níveis de enzimas antioxidantes. Entretanto, embora esses resultados sejam significativos, Pinho e colaboradores ${ }^{12}$ sugerem que o efeito do treinamento físico sobre a redução nos níveis de lipoperoxidação não é tão significativo quanto seus efeitos sobre a melhora na capacidade do tecido em resistir aos danos oxidativos gerados por radicais livres. É possível que essa melhora na resistência do tecido explique o fato do treinamento não diminuir os níveis de lipoperoxidação, mas impedir que esses níveis aumentassem.

Similarmente aos resultados obtidos na lipoperoxidação, a exposição à BLM elevou, significativamente, os níveis de carbonilação de proteínas, o que nesse caso específico, o treinamento preventivo e terapêutico não reduziu esses efeitos, conforme os resultados mostrados na figura 3. As ERO podem modificar aminoácidos por reações em cadeias através de agregados de proteínas suscetíveis a degradações proteolíticas. Durante esse processo, alguns aminoácidos são convertidos em derivados de carbonil ${ }^{10}$.

Outros estudos de nosso laboratório têm mostrado diminuições dos níveis de carbonilação em pulmão de roedores após o treinamento físico ${ }^{13,27,31}$. Segundo Pinho e colaboradores ${ }^{13}$, é possível que essas diferenças em estudos possam estar relacionadas a quatro aspectos: primeiro, o uso ou não e os tipos de agentes estimuladores de danos que podem apresentar repostas diferenciadas induzidas pelo exercício físico; segundo, a metodologia utilizada para determinar os níveis de oxidação protéica; terceiro, o tipo, duração, frequência e intensidade do exercício utilizado; quarto, é possível, ainda, que essas diferenças estejam relacionadas à redução na taxa do turnover de proteínas. Sobre este último, está bem estabelecido na literatura que as proteínas modificadas ou oxidadas são menos degradadas por proteossomas. Isso decorre do aumento na atividade de proteossomas como um processo adaptativo secundário à oxidação de proteínas após o exercício ${ }^{28,33}$. Embora ainda seja uma hipótese, pode ser a principal justificativa para as diferenças encontradas em diversos estudos, como também, pode justificar o aumento na carbonilação nos grupos salina submetidos a ambos os programas de treinamento.

A atividade da SOD e Catalase no tecido reflete, parcialmente, o sistema de defesa enzimático do pulmão contra a produção de radicais livres. Os resultados observados na figura 4 mostram uma variação na atividade da SOD entre os grupos treinados. Inicialmente, o treinamento preventivo provocou um aumento significativo na atividade da SOD no grupo tratado com BLM, enquanto que o exercício terapêutico diminuiu significativamente esses valores em relação ao grupo salina.

O treinamento físico aeróbio melhora o sistema enzimático de ação antioxidante, protegendo a células contra a produção excessiva de radicais livres $^{34}$. Entretanto, esses efeitos estão relacionados às características do exercício ou do treinamento ${ }^{12}$.

Os resultados observados na atividade da SOD sugerem um efeito preventivo do exercício sobre a lesão pulmonar. Segundo Bowler ${ }^{30}$ é possível que a secreção de colágeno por fibroblastos seja estimulada pelo ânion superóxido. Assim, o aumento na atividade da SOD induzida pelo exercício reduz esse efeito, protegendo o tecido pulmonar. Portanto, antioxidantes como a SOD, tem um importante papel no sistema de defesa pulmonar contra a lesão causada pela BLM. Este fato pode justificar, também, o não aumento nos danos oxidativos observados na figura 2 e 3 . Adicionalmente, é possível que o exercício físico também melhore a micro-circulação pulmonar e diminua a resposta inflamatória primária, impedindo a liberação de fatores pró-fibrogênicos ${ }^{12}$.

Por outro lado, o treinamento físico (terapêutico) não aumentou a atividade da SOD após a lesão pulmonar. Esta resposta pode estar associada com a adaptação do tecido pulmonar à lesão provocada pela BLM. E ainda, à diminuição do processo fibrogênico observados na figura 1.

Diferenciadamente do observado na atividade da SOD, a atividade da CAT apresentou valores 
significativamente menores após ambos os programas de treinamento, tanto no grupo salina quanto no grupo tratado com BLM (figura 5).

A CAT é uma enzima que cataliza a degradação do peróxido de hidrogênio ${ }^{10}$ e, portanto, sua atividade depende do produto gerado a partir da dismutação do ânion superóxido. No treinamento terapêutico, a baixa atividade da SOD, no grupo tratado com BLM (figura 4), pressupõe uma baixa formação de peróxido de hidrogênio o que também sugere uma atividade da CAT diminuída. Entretanto, no grupo que treinou preventivamente, os resultados da SOD e CAT são inversos. É possível que, nesse caso, outros sistemas de defesa enzimáticos estejam, predominantemente, favorecendo catálise do peróxido de hidrogênio, como a glutationa peroxidase.

\section{CONCLUSÃO}

Com base nos resultados apresentados, sugerimos que o treinamento preventivo e ou terapêutico possa ser uma boa alternativa na prevenção e tratamento do dano pulmonar induzidos por agentes químicos, como também, melhorar os mecanismos de defesa pulmonar contra os danos causados por estresse oxidativo. Contudo, novos estudos são necessários para elucidar as diferenças encontradas nos mecanismos de defesas antioxidantes pulmonar induzidos tanto pela exposição à BLM quanto pelo exercício físico realizado de forma preventiva ou terapêutica.

\section{REFERÊNCIAS BIBLIOGRÁFICAS}

1. Borzone G, Moreno R, Urrea R, Meneses M, Oyarzún M, Lisboa C. Bleomycin-induced chronic lung damage does not resemble human idiopathic pulmonary fibrosis. Am J Respir Crit Care Med 2001;163(7): 1648-1653.

2. Cortijo J, Cerda-Nicola M, Serrano-Mollar A, Bioque G, Estrela JM, Santangelo F, et al. Attenuation by oral $\mathrm{N}$-acetylcysteine of bleomycin-induced lung injury in rats. Eur Respir J 2001;17(6):1228-1235.

3. Serrano-Mollar A, Closa D, Prats N, Blesa S, MartinezLosa M, Cortijo J et al. In vivo antioxidant treatment protects against bleomycin-induced lung damage in rats. Br J Pharmacol 2003;138(6):1037-1048.

4. Mata M, Ruíz A, Cerda M, Martinez-Losa M, Cortijo J, Santangelo F et al. Oral N-acetylcysteine reduces bleomycin-induced lung damage and mucin Muc5ac expression in rats. Eur Respir J 2003;22(1):900-905.

5. Yildirim Z, Kotuk M, Iraz M, Kuku I, Ulu R, Armutcu F, Ozen S. Attenuation of bleomycin-induced lung fibrosis by oral sulfhydryl containing antioxidants in rats: erdosteine and $\mathrm{N}$-acetylcysteine. Pul Pharmacol Ther 2005;18(5):367-373.

6. Bowden DH. Unraveling pulmonary fibrosis: the bleomycin model. Lab Invest 1984;50(5):487-488.
7. Hay J, Shahzeidi S, Laurent G.Mechanisms of bleomycin-induced lung damage. Arch Toxicol 1991;65(2):81-94.

8. Tsuda E, Goto M, Mochizuki S, Yano K, Kobayashi F, Morinaga T, Higashio K. Isolation of a novel cytokine from human fibroblasts that specifically inhibits osteoclastogenesis. Biochem Biophys Res Commun 1997; 234(1):137-142.

9. Stevenson CS, Koch LG, Britton SL. Aerobic capacity, oxidant stress, and chronic obstructive pulmonary disease - A new take on an old hypothesis. Pharmacol Ther 2006;110(1):71-82.

10. Halliwell B, Gutteridge JMC. Free Radical in Biology Medicine University Press, Oxford, NY, 2007.

11. Hatao H, Oh-ishS, Itoh M, Leeuwenburgh C, Ohno H, Ookawara T, Kishi K, Yagyu H, Nakamura H, Matsuoka T. Effects of acute exercise on lung antioxidant enzymes in young and old rats. Mech Ageing Dev 2006; 127(4):384-390.

12. Pinho RA, Chiesa D, Mezzomo KM, Andrades ME, Bonatto F, Gelain D, Dal Pizzol F, Knorst MM, Moreira JC.Oxidative stress in chronic obstructive pulmonary disease patients submitted to a rehabilitation program. Respir Med 2007; 101(8):1830-1835.

13. Pinho, RA, Silva, GA, Silveira, PCL, Tuon, T, DalPizzol, F, Moreira, JCF. Exercício físico regular diminui o estresse oxidativo pulmonar em ratos apos exposição aguda ao carvão mineral. Rev Bras Med Esporte 2006; 12(2):81-85.

14. König D, Berg A. Exercise and oxidative stress: Is there a need for additional antioxidants. Österreichisches J Für Sportmedizin 2002;3(1):6-15.

15. Gomez-Cabrera M-C, Domenech E, Viña J. Moderate exercise is na antioxidant: upregulation os antioxidant genes by training. Free Radic Biol Med 2008;44(2):126-131.

16. Ji LL. Modulation of skeletal muscle antioxidant defense by exercise: role of redox signaling. Free Radic Biol Med 2008;44(2):142-152.

17. Olert ED, Cross BM, McWillians AA. Guide to care and use of experimental animals. 2th ed. Ottawa: Canadian Council on Animal. 1993.

18. Fernando P, Bonen A, Hoffman-Goetz L. Predicting submaximal oxygen consumption during treadmill running in mice. Can J Physiol Pharmacol 1993;71(10):854-857.

19. Woessner JB. The determination of hidroxyproline in tissue and protein samples containing small proportions of this amino acid. Arch Biochem Biophysics 1961;93(1):440-447.

20. Draper HH, Hadley M. Malondialdehyde determination as index of lipid peroxidation. Meth Enzymol 1990;186 (1):421-431.

21. Levine RL, Garland D, Oliver CN, Amici A, Climent I, Lenz AG, et al. Determination of carbonyl content in oxidatively modified proteins. Meth Enzymol 1990;186(1):464-478.

22. Bannister JV, Calabrese L. Assays for SOD. Meth Bioch Analyt 1987; 32(2):279-312.

23. Aebi H. Catalase in vitro. Meth Enzymol 1984;105(1):121-126.

24. Lowry OH, Rosebough NG, Farr AL, Randall RJ. Protein measurement with the folin phenol reagent. J Biol Chem 1951;193(1):265-275. 
25. Hagiwara S, Ishii Y, kitamura S. Aerosolized administration of $\mathrm{N}$-acetylcysteine attenuates lung fibrosis induced by bleomycin in mice. Am J Respir Crit Care Med 2000;162(1):225-231.

26. Serrano-Mollar A, Closa D, Cortijo J, Morcillo EJ, Prats N, Gironella M et al. P-selectin upregulation in bleomycin induced lung injury in rats: effect of $\mathrm{N}$. acetyl-L-cysteine. Thorax 2002;57(7):629-634.

27. Teixeira KC, Soares FS, Rocha LG, Silveira PC, Silva LA, Valença SS, Pizzol FD, Streck EL, Pinho RA.Attenuation of bleomycin-induced lung injury and oxidative stress by $\mathrm{N}$-acetylcysteine plus deferoxamine. Pulm Pharmacol Ther 2008;21(2):309-316.

28. MacNee W, Selby C. Neutrophil traffic in the lungs, the role of hemodynamics, cell adhesion and deformabilty. Thorax 1993;48(1):79-88.

29. Sime PJ, O'Reilly KM. Fibrosis of the lung and other tissues: new concepts in pathogenesis and treatment. Clin Immunol 2001;99(3):308-319.

30. Bowler RP, Nicks M, Warnick K, Crapo JD. Role of extracellular superoxide dismutase in bleomycin-induced pulmonary fibrosis. Am J Physiol Lung Cell Mol Physiol 2002;282(4):719-726.

31. Pinho RA, Silveira PC, Silva LA, Streck EL, Dal-Pizzol F, Moreira JCF. N-acetylcysteine and deferoxamine reduce pulmonary oxidative stress and inflammation in rats after coal dust exposure. Environ Res 2005;99(3):355-360.
32. Iraz M, Erdogan H, Kotuk M, Yagmurca M, Kilıc T, Ermis H, Fadillioglu E, Yildirim Z. Ginkgo biloba inhibits bleomycin-induced lung fibrosis. Pharmacol Res 2006;53(3):310-336.

33. Grune T, Reinheckel T, Davies K J. Degradation of oxidized proteins in mammalian cells. FASEB J 1997;11(7):526-534.

34. Leaf DA, Kleinman MT, Hamilton M, Deitrick R. The exercise-induced oxidative stress paradox: the effects of physical exercise training. Am J Med Sci 1999;317(5):295-300.

\section{Agradecimentos}

Nossos agradecimentos aos órgãos financiadores deste estudo: CAPES, CNPq e UNESC.

\section{Endereço para correspondência}

Ricardo Aurino de Pinho

Laboratório de Fisiologia e Bioquímica

do Exercício/UNESC

Av. Universitária, 1105 - Bairro Universitário

88806-000 - Criciúma - SC/Brasil

E-mail: pinho@unesc.net 